\title{
Prácticas educativas mediadas por TIC. Un análisis de la metodología del profesorado a través del Programa Escuela 2.0
}

\author{
Carlos José González Ruiz. Universidad de La Laguna \\ Sebastián Martín Gómez. Universidad de La Laguna
}

0000-0003-4269-6221

0000-0002-3367-6221

Recepción: 11/10/2019 | Aceptado: 25/11/2019

Correspondencia a través de ORCID: Carlos José González Ruiz

iD 0000-0003-4269-6221

Citar: González, J y Martín, S (2019). Prácticas educativas mediadas por TIC. Un análisis de la metodología del profesorado a través del Programa Escuela 2.0. REIDOCREA, 8(2), 75-85.

\begin{abstract}
Resumen: Las estrategias metodológicas llevadas a cabo por el docente en el aula llevan siendo objeto de estudio durante las últimas décadas. La inclusión de las TIC en el aula ordinaria ha revolucionado de una manera u otra la metodología usada por el profesorado. Un ejemplo de ello fue el Programa Escuela 2.0, que nació con el objetivo de digitalizar el sistema educativo español a través de la dotación de recursos: mini-portátiles, Pizarras digitales interactivas y conexión a internet. En este artículo, y con la inestimable colaboración de cuatro centros educativos públicos de educación primaria, analizamos la llegada del programa mencionado con anterioridad, a través de la metodología de estudio de casos, centrándonos en las estrategias metodológicas llevadas a cabo por los docentes. Para ello, se han realizado doce entrevistas al equipo directivo y profesorado de los centros, se han observado prácticas de aula, así como se ha hecho un análisis de dichos datos para poder ofrecer un panorama más actual. Los resultados nos indican que la propia llegada de dichos recursos al aula ha hecho que el profesorado se replantee su dinámica de clase, pues antes se centraba única y exclusivamente en el libro de texto.
\end{abstract}

Palabras clave: Estrategias de enseñanza | TIC

ICT-mediated educational practices. An analysis of the teaching staff methodology through the Escuela 2.0 program

\begin{abstract}
The methodological strategies carried out by the teacher in the classroom have been the object of study during the last decades. The inclusion of ICT in the ordinary classroom has revolutionized in one way or another the methodology used by teachers. An example of this was the Escuela 2.0 Program, which was born with the objective of digitizing the Spanish education system through the provision of resources: mini-laptops, interactive whiteboards and internet connection. In this article, and with the invaluable collaboration of four public primary education centers, we analyze the arrival of the aforementioned program, through the case study methodology, focusing on the methodological strategies carried out by teachers. For this, twelve interviews with the management team and teachers of the centers have been carried out, classroom practices have been observed, as well as an analysis of said data to offer a more current panorama. The results indicate that the very arrival of these resources in the classroom has led teachers to rethink their class dynamics, as before they focused solely and exclusively on the textbook.
\end{abstract}

Keywords: Teaching strategies | ICT

\section{Introducción}

Hace ya diez años que Hargreaves (1999) afirmaba que los tiempos estaban cambiando y por lo tanto el profesorado tendrá que adaptarse a dichos cambios. La aparición de las TIC, y más concretamente de la segunda generación de la web hacía comenzar a replantearse cómo la tecnología afectaría a la educación como se conocía hasta entonces.

En torno al año 2004 se usaba ya de forma estandarizada el término de Web 2.0, y con éste comenzaban a diferenciarse las primeras ventajas de los sistemas digitales aplicados a la enseñanza. Podemos encontrar en el trabajo de García (2014) una clasificación de estas características: 
- Interactividad entendida como la posibilidad de una comunicación bidireccional y multidireccional, tanto de forma síncrona como asíncrona.

- Accesibilidad. Facilidad de acceder a una gran cantidad de recursos (documentos de texto en su mayoría en el momento en el que se empezaba a usar el término).

- Aprendizaje colaborativo. Aprender con otros y de otros, y poder desarrollar un aprendizaje de forma guiada, así como acceder a la información en el mismo momento por varios usuarios.

- Libertad de edición y difusión. Difundir los trabajos realizados, las ideas planteadas entre una comunidad ampliada, y que estos trabajos fueran a su vez usados por otros usuarios siendo reeditados.

Entre estas características propias de la Web, como es el acceso a los contenidos, la interconexión (hipertexto), y la posibilidad para la creación y edición, sería esta última, la creación, la que se vio más potenciada con la 2.0.

Desde el punto de vista del aula, la Web 2.0 se entendía como el paso de la web únicamente destinada a la lectura a ser un espacio de lectura y escritura (De la Torre, 2006, Area y Pessoa, 2012). Este hecho tuvo como exponente a los primeros blogs o edublogs. Una forma fácil, accesible y gratuita de poder escribir tanto individualmente o colaborativamente en la red. Su uso se estandarizó a medida que los primeros ordenadores llegaban a las aulas de forma masiva, creando tanto blogs de asignaturas gestionados por los propios docentes a modo de entorno virtual en el que compartir trabajos de sus alumnos; como blogs individuales o grupales del alumnado a modo de portafolio. Este es solo un ejemplo de las prácticas de aula desarrolladas con estrategias metodológicas apoyadas por TIC que se vieron potenciadas.

En España este movimiento se vio precedido por la implantación de políticas educativas que pretendían introducir la tecnología en los centros educativos. En concreto, a nivel estatal, la gran apuesta fue el Programa Escuela 2.0, el cual describiremos en las siguientes páginas. A su vez, presentaremos resultados de un estudio realizado en diferentes centros educativos, en el que se analiza la visión de los docentes frente a la incorporación de las nuevas metodologías de aula apoyadas con TIC que derivaron de dicho programa.

\section{De la Web 2.0 al Programa Escuela 2.0}

Como ya hemos introducido, la integración de la tecnología en las aulas en España se vio maximizada por su gran apuesta, el Programa Escuela 2.0. Sin embargo, la preocupación de incorporar las TIC a los centros educativos españoles cuenta con una larga trayectoria. Como bien expone Area (2014), durante los años 80 se dieron los primeros intentos por modernizar las aulas con diversas políticas educativas impulsadas por el gobierno central español. Sin embargo, estas políticas desaparecieron al poco tiempo con la consolidación de los diversos gobiernos autonómicos. En este cambio, muchas Comunidades Autónomas, con un poder en materia educativa, desarrollaron programas y acciones a nivel regional para llevas las TIC a los centros escolares. Estas políticas, aunque siguieran un patrón y directrices marcadas por la Unión Europea al contar con su financiación, se llevaron a cabo de forma separa y paralelamente, sin existir un plan nacional que abordase esta cuestión en todo el estado español.

Estos planes regionales se vieron interrumpidos con la llegada de un nuevo proyecto, esta vez sí consensuado para todo el territorio nacional, que tuvo una duración corta comprendida entre el 2009 y el 2012. Este nuevo plan se presentó con el nombre de Programa Escuela 2.0. Los principales objetivos de dicho proyecto fueron: 
- La transformación en aulas digitales de todas las aulas de $5^{\circ}$ y $6^{\circ}$ de Educación Primaria y de $1^{\circ}$ y $2^{\circ}$ de Educación Secundaria Obligatoria de los centros públicos.

- La dotación de ordenadores para el uso personal de todos los alumnos de los mencionados cursos, matriculados en centros sostenidos con fondos públicos, en proporción 1 a 1.

- La realización de acciones de formación de profesorado para garantizar el uso eficaz de los recursos del programa.

- El desarrollo de contenidos digitales que pudieran ser utilizados por los docentes.

Esta propuesta se vio sumergida en un despunte del movimiento por digitalizar las aulas escolares a nivel mundial. El Programa Escuela 2.0 seguía los pasos de los primeros proyectos iniciados durante la primera década del siglo XXI, donde la tecnología ya cada vez tenía más presencia (Area, 2014). Estas políticas nacionales optaron por desarrollar el denominado <<modelo 1:1 >>. Este modelo, extendido en muchas escuelas y usado ahora en sistemas escolares de países de todo el mundo, guarda la proporción de "un ordenador por alumno/a". La implantación de este modelo y de los dispositivos en las aulas base de los centros escolares, llevó consigo no solo un aumento de tareas o actividades mediadas por ordenador por parte del alumnado, sino que supuso en algunos casos un cambio el inicio en la metodología docente. Las oportunidades que brindaban estos nuevos equipos y la disponibilidad de que cada alumno pudiera trabajar con su ordenador; hicieron que muchos docentes se replanteasen sus prácticas de aula habituales.

\section{Estrategias pedagógicas con TIC}

Area (2008), clasifica en su revisión de la innovación pedagógica con TIC y cómo esta se relaciona con el desarrollo de las competencias informacionales y digitales, las que pueden ser las principales tareas o acciones que lleva implícito el uso de la tecnología en el aula:

1. El apoyo a las exposiciones del docente. El Pizarras Digitales Interactivas y las presentaciones multimedia son un estándar en cualquier aula digital.

2. Demanda de actividades interactivas. Gran parte de las tareas demandadas a alumnado se realizan mediante algún software concreto presente el dispositivo. En la mayoría de casos, estas tareas precisan de conexión a internet para acceder a entornos virtuales o aplicaciones donde se han de desarrollar las tareas.

3. Ampliación de los contenidos de materiales impresos. Actualmente muchos centros educativos en España se encuentran en proceso de dejar de lado el libro de texto como material de estudio único. Los recursos analógicos y digitales conviven en las aulas, complementándose mutuamente en muchos casos. El desarrollo de metodologías como el aprendizaje basado en proyectos (ABP) requieren que el alumno tenga la autonomía y tecnología necesaria para hacer búsquedas en Internet, realizar investigaciones, extraer datos, sintetizar información, etc.

4. Manejo de software y desarrollo de la competencia digital. Lógicamente, todas las tareas que se desarrollan mediante los dispositivos o recursos digitales en las aulas llevan consigo el desarrollo de la competencia digital en el alumnado. 


\section{Objetivo general}

Analizar la metodología didáctica con uso de las TIC a partir de los recursos proporcionados por el programa REDXXI/Escuela 2.0

\section{Preguntas de investigación}

¿Cómo afecta la aplicación del programa Escuela 2?0/REDXXI en el profesorado participante en relación con las estrategias didácticas?

¿Han cambiado las prácticas educativas del profesorado usando las herramientas proporcionadas por el programa Escuela 2?0/REDXXI? ¿Qué uso de la WEB 2.0 realizan los participantes del programa?

\section{Método: Estudio de Caso}

El método escogido para el desarrollo de dicho trabajo ha sido Estudio de caso. Existen varias razones del porqué se ha escogido dicha metodología que mostraremos a continuación:

- Stake (1999) nos dice que los casos que son de interés en la educación lo constituyen en su mayoría personas y programas. En esta ocasión nos encontramos con un programa educativo de integración de las TIC (REDXXI/Escuela 2.0) en el que participarán personas, con lo que se adecua perfectamente a nuestros objetivos.

- La elección de dicho método ha sido la necesidad de realizar una investigación en un contexto real. Se hace necesario poder acceder a los usos y prácticas pedagógicas con ordenadores que hacen los sujetos educativos en las aulas escolares, ya que el principal objetivo de dicho trabajo es conocer las estrategias metodológicas llevadas a cabo por el profesorado participante en dicho programa.

\section{Procedimiento y estrategia metodológica}

\begin{tabular}{|c|c|c|}
\hline Dimensión & Variables/Indicadores & Instrumentos \\
\hline $\begin{array}{l}\text { Enseñanza } \\
\text { (Metodología } \\
\text { didáctica) }\end{array}$ & $\begin{array}{l}\text { Estrategias de enseñanza } \\
\text { Facilitadores de aprendizaje (motivación, } \\
\text { interacción, etc.). }\end{array}$ & $\begin{array}{l}\text { Entrevista equipo } \\
\text { directivo. } \\
\text { Entrevista profesorado. } \\
\text { Observación. }\end{array}$ \\
\hline
\end{tabular}

\section{Contexto/Participantes}

Debido a las características de la investigación y a la decisión metódica escogida (estudio de casos), se utiliza un muestreo intencionado para aumentar la utilidad de la información obtenida a partir de pequeños modelos, así como con la intención de que sean inteligibles e informativos sobre los fenómenos que el investigador está investigando (McMillan, Schumacher, 2005). 
Se hace necesario escoger aquellos centros que respondan a las preguntas y objetos de investigación de dicho trabajo. Simons (2011) comenta que hay que tener en cuenta varios factores a la hora de elegir un caso:

- El tipo de estudio de caso que se desea realizar.

- ¿Dónde está ubicado el caso?

- ¿Qué es lo que va a generar mayor comprensión?

- Los costes de desplazamiento.

- El tiempo que haya que emplear

El procedimiento llevado a cabo para la selección de la muestra de dicho trabajo de investigación fue el siguiente:

- La selección de la muestra se ha llevado a cabo sobre distintos centros pertenecientes a los cursos $5^{\circ}$ y $6^{\circ}$ de primaria ubicados en el distrito universitario de Salamanca (Ávila, Zamora, Salamanca) participantes en el Programa Escuela 2.0 (REDXXI).

- Se ha establecido una comunicación con todos los centros, vía web o presencial del estudio que estamos realizando.

- Revisión de todos los centros del distrito universitario de Salamanca (Ávila, Zamora, Salamanca) que hayan recibido toda la dotación de recursos del programa REDXXI/Escuela 2.0.

- Tras esta revisión hemos seleccionado los centros que consideremos más adecuados para llevar a cabo la realización del trabajo y que han estados dispuestos a colaborar con la intención de mejorar su práctica pedagógica.

- Después de dicha revisión se han escogido cuatro centros de primaria ubicados en el distrito universitario de Salamanca. CEIP Francisco de Vitoria y CEIP Juan Jaén (Salamanca). CEIP Obispo Nieto (Zamora) y CEIP Comuneros de Castilla (Ávila)

Al tratarse de un estudio de casos instrumental se puede escoger cualquiera de una serie de casos para investigar el tema que se está estudiando (Simons, 2011). En esta ocasión el principal criterio por lo que se ha escogido a los centros educativos nombrados con anterioridad es muy claro: haber recibido la dotación de los recursos proporcionados por el Programa Escuela 2.0/REDXXI, ya que eran los únicos que podrían responder a las preguntas de investigación nombradas con anterioridad.

Los centros educativos que han querido participar en dicha investigación muestran una serie de aspectos en común que son necesarios explicitar:

- Son centros educativos de infantil y primaria públicos pertenecientes a la provincia Universitaria de Salamanca, habiendo dos centros ubicados en el centro de la provincia de Salamanca, uno en el centro de la provincia de Zamora y otro en el centro de la provincia de Ávila.

- Todos los centros participantes han recibido la dotación del Programa Escuela 2.0, esto es: Pizarra digital, conectividad, así como el armario en dónde se encuentran los mini-portátiles para cada alumno. 
Instrumentos de recogida de información

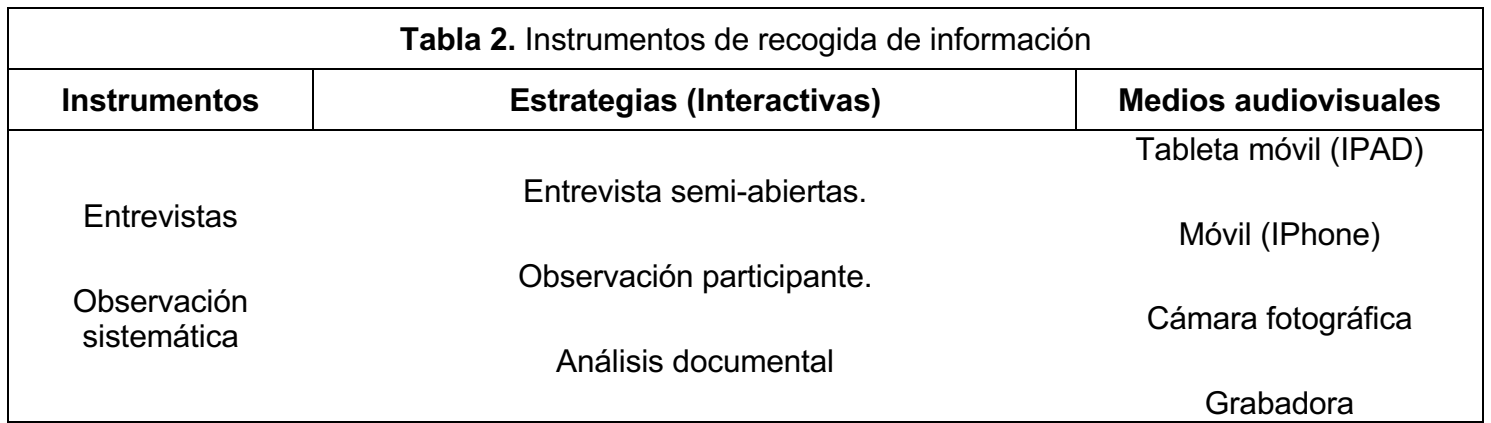

Las entrevistas se realizaron al equipo directivo de los cuatro centros seleccionados, así como al profesorado participante en el Programa Escuela 2.0, que en este caso pertenecían a las etapas de $5^{\circ}$ y $6^{\circ}$ de Primaria. El principal objetivo de dichas entrevistas fue recopilar la información suficiente sobre los distintos aspectos del Programa, en especial, sobre las estrategias metodológicas llevadas a cabo por el profesorado.

En cuanto a las observaciones se realizaron dieciséis observaciones de aula correspondientes a las etapas de $5^{\circ}$ y $6^{\circ}$ de Primaria. En todas ellas, se observó por parte de los investigadores el uso que hacían el profesorado y alumnado de los recursos proporcionados por el Programa Escuela 2.0/REDXXI. El principal objetivo de dichas observaciones era comprobar de manera directa y práctica, las diferentes estrategias metodologías del profesorado, para de esta manera, poder corroborar, la información proporcionada a través de las entrevistas realizadas.

\section{Análisis de datos}

Con respecto al análisis de la información obtenida a través de los instrumentos de información, procedemos a mostrar el procedimiento realizado.

\section{Análisis de las entrevistas}

Se llevaron a cabo el análisis sobre diferentes entrevistas realizados al profesorado de $5^{\mathrm{a}}$ y $6^{\mathrm{a}}$ de Primaria, así como al equipo directivo de los centros educativos analizados:

- Entrevistas al profesorado: se llevaron a cabo ocho entrevistas, una al profesor de $5^{a}$ de primaria y otra al de $6^{a}$ de Primaria de todos los centros educativos.

- Entrevistas al equipo directivo: se llevaron a cabo cuatro entrevistas, correspondientes a cada uno de los centros educativos.

- Número total de entrevistas: 12.

El análisis de las entrevistas se llevó a cabo a través del programa cualitativo de datos "Atlas.ti". Para ello se llevó a cabo el siguiente procedimiento:

- Realización de las entrevistas.

- Transcripción de las entrevistas en documento de texto.

- Subida de los documentos al programa cualitativo Atlas.Ti.

- Codificación de la información, la cual consiste en identificar trozos de texto y descubrir que códigos representan desde un punto de vista teórico y analítico.

- Creación de categorías en función de la codificación realizada con anterioridad. 
Después de realizar dicho procedimiento se llevó a cabo un profundo análisis de los datos en relación a la variable a investigar, en este caso: las estrategias metodológicas llevadas a cabo por el profesorado en relación a los recursos proporcionados por el Programa Escuela 2.0/REDXXI. En la Figura 1 podemos observar una nube de palabras en dónde podemos identificar aquellas categorías (códigos) más nombrados en las entrevistas realizadas.

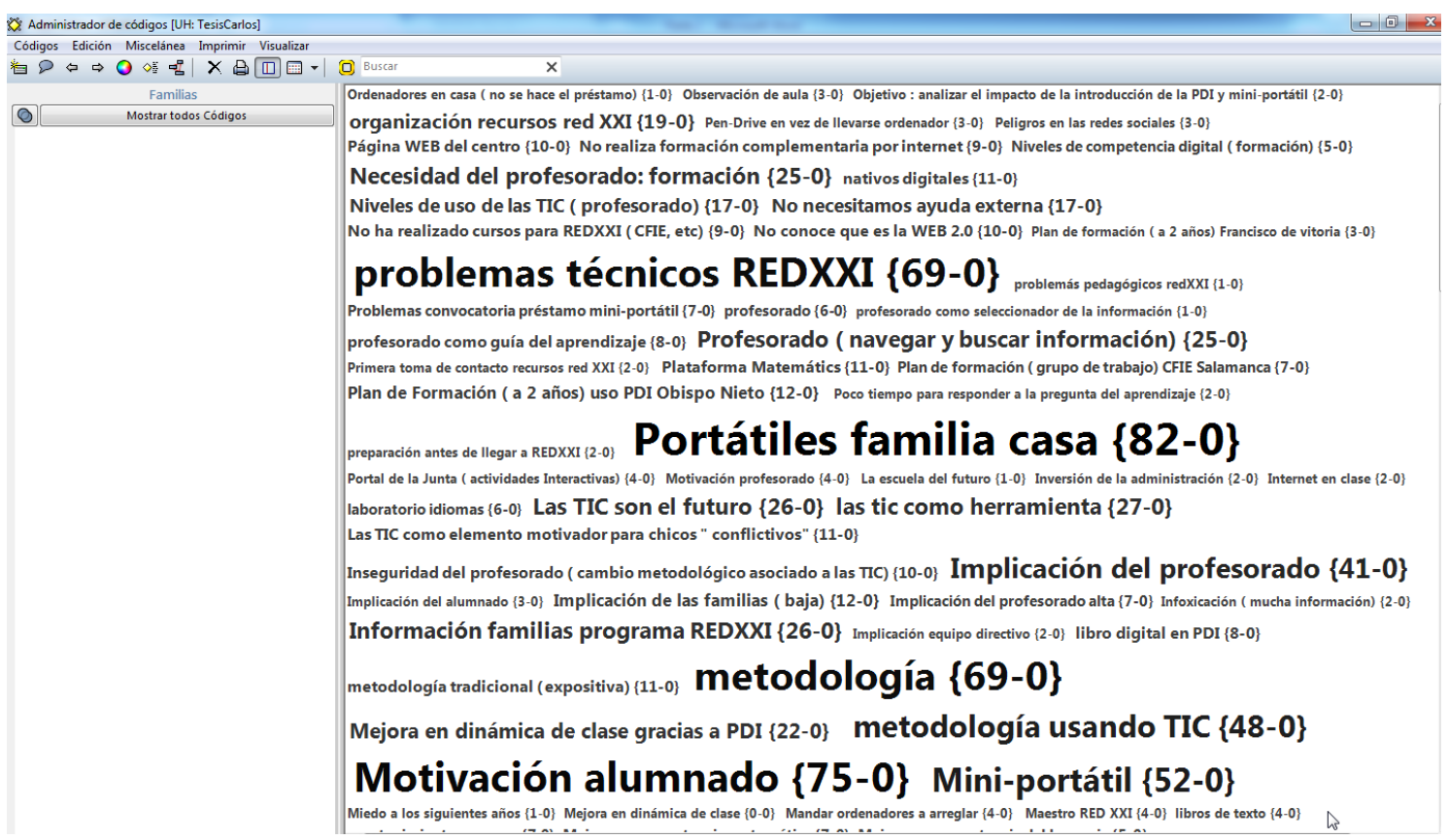

Figura 1. Mapa de nube de las categorías creadas con el programa Atlas.ti

\section{Resultados}

Todos los maestros y maestras de los centros educativos afirman que con el uso de dichos recursos proporcionados por el programa REDXXI/Escuela 2.0, su forma de dar clases, está cambiando. Aun afirmando que las TIC es una herramienta más, creen que el trabajar por competencias, y la vez, hacer uso de dichas herramientas, propiciará un cambio con total seguridad. Aquellos con más experiencia creen que es una situación que requerirá tiempo pero que es inevitable.

En el CEIP Comuneros de Castilla (Ávila) y CEIP Francisco de Vitoria (Salamanca) están completamente convencidos de que su forma de dar clases ha cambiado con la llegada de los recursos proporcionados por el programa. Se destaca el trabajo autónomo por parte del alumnado, y cómo esta situación ha hecho replantear los roles educativos en sus aulas. En el CEIP Comuneros se han llegado incluso a plantear la eliminación de algún libro de texto y en el CEIP Francisco de Vitoria creen que es una herramienta fantástica para acercar la realidad al alumnado.

Mucho, bastante cambio de metodología y además más activa. Y no llevas a los niños igual, cada uno va de distinto, son más independientes. Tu papel en algunos momentos ha cambiado un poco con respecto a antes que eras tú el que dabas todas las instrucciones, es un poco como de coordinador. Además, ellos mismos son conscientes de su auto-aprendizaje, les proporcionas unas herramientas para ellos mismos buscarse sus estrategias para aprender, antes 
eras tú el que le estabas diciendo, esto, lo otro...ahora ellos mismos van viendo... (Maestro 5 de primaria del CEIP Comuneros de Castilla)

Cuando vine me daba miedo coger sexto por el tema de REDXXI, ordenadores, PDI. Ahora estoy encantada con el sexto, he ido poco a poco, he aprendido cosas, he cambiado mi manera de trabajar en el aula... (Maestra de 6 de primaria del CEIP Francisco de Vitoria)

En el CEIP Obispo Nieto (Zamora) existe disparidad de opiniones, según el maestro al que se le pregunte. Aunque todos afirman que existe un cambio, la maestra más joven del centro cree que ya está afectando su manera de dar clases, mientras que el coordinador TIC y también maestro participante del programa REDXXI/Escuela 2.0 cree que es un proceso lento y que no sabe si él se va adaptar a las exigencias de cambio metodológico, que desde diferentes instancias se le pide al profesorado.

Es que es diferente completamente. Te obliga a trabajar de otra manera. Ellos son a la vez más independientes, al principio están todo el día levantando la mano diciendo: esto no me sale, esto..., pero después que ya han cogido la dinámica como que son más independientes. También por ejemplo a la hora de escribir en el foro, o que tengan que subir documentos a la red...es distinto. (Maestra de 5 de primaria del CEIP Obispo Nieto)

En el CEIP Juan Jaén (Salamanca) encontramos mucha disparidad también de opiniones y prácticas educativas. El director del centro insiste en la importancia de utilizar dichos recursos de manera distinta a cómo usaban los recursos anteriores. Sin embargo, la edad avanzada de muchos de los maestros, así como la dificultad del cambio de concepción de las prácticas educativa, está dificultando este proceso.

$\mathrm{Si}$, además es que yo he tenido que cambiar todas mis programaciones, Carlos. No solamente porque la ley también nos obliga. Las competencias, la competencia TIC es fundamental, entonces yo sí que me he visto obligada gustosamente a cambiar mi metodología. (Maestra 6 de primaria del CEIP Juan Jaén)

Otra de las cuestiones que destaca la mayoría del profesorado es el acceso a la información que tienen desde el aula, tanto con la Pizarra Digital Interactiva como con los mini-portátiles. Esto hace que en multitud de ocasiones se interrumpa la clase para hacer uso de dicho recurso y ampliar la información que en ese momento trabajan.

\section{Resultados en relación al aprendizaje colaborativo}

Otros de los resultados importantes a destacar se relaciona con el aprendizaje colaborativo del alumnado realizado a través de una de los recursos proporcionados por el Programa Escuela 2.0/REDXXI: los mini-portátiles. A continuación, podremos ver diferentes ejemplos a través de las entrevistas realizadas al profesorado.

$\mathrm{Si}$, muchísimo. A través de Edmodo se comunican entre ellos todos los días y muy a menudo. Se preguntan los deberes. Por ejemplo falta alguien y se preguntan, oyes no has venido, ¿Cómo estás? ¿Qué está pasando? La verdad es que ahora se llevan bastante bien entre ellos, se ayudan en tareas de clase y además muchas veces quedan a través de Edmodo que los veo yo, bien, bien (Maestra del CEIP Juan Jaén). 
Si se nota que son más colaboracionistas. A mí me ha sorprendido una alumna como le da por mensajería instrucciones a otra compañera para entrar en el foro: <primero entras, luego buscas tu libro, luego donde pone no sé qué y empiezas a escribir...">, parece un tutorial escrito. Son capaces de ayudarse, es colaborativa en ese aspecto (Maestro del CEIP Obispo Nieto).

El hecho de que yo pudiera desde casa mandarles a ellos todo eso que yo quería darles en la hora de clase me permitió a mi entrar ahí y ver lo valioso que era. $Y$ ahora que ellos puedan mandarme a mí. Hoy me ha dicho una niña: "te he mandado por Edmodo una actividad que he hecho sobre las fracciones que es lo que estamos trabajando ahora". Me la tengo que ver esta noche, pero me la veo ilusionada. Tenemos un nivel de contacto tan grande... (Maestra del CEIP Francisco de Vitoria).

"Claro que potencia, evidentemente. Es vital para el grupo de alumnos que tenemos, entonces entre ellos mismos colaboran a través de las TIC. Y no solo el colaborativo, el significativo y el constructivo también (Maestro del CEIP Comuneros de Castilla).

El profesorado de los centros educativos afirma que su metodología está cambiando, que se necesita un tiempo para adaptarse, pero están convencidos que el uso de dichas herramientas ha modificado su forma de dar clases.

\section{Discusión y conclusiones}

Las prácticas pedagógicas más usuales observadas no son muy relevantes y se relacionan con las expuestas por Area (2008), y que reproducimos a continuación.

Tabla 3. Prácticas pedagógicas más observadas

- Apoyar las exposiciones magistrales del profesor en el aula.

- Demandar al alumnado la realización de tareas interactivas y repetitivas con un grado de complejidad muy bajo.

- Ampliar los contenidos del libro a través de búsquedas realizadas en Internet.

- Enseñar al alumnado competencias informáticas a través del uso de algún programa informático.

Con respecto a las diferentes formas de aprender del alumnado se destacan diferentes estrategias didácticas de enseñanza llevadas a cabo por el profesorado con los recursos proporcionados por el programa REDXXI. El uso de dichas herramientas ha posibilitado las diferentes estrategias en el aula y fuera de ella. 
Tabla 4. Estrategias didácticas más significativas

- Aprendizaje autónomo: posibilidad de que el alumnado trabaje de forma individual y autónoma. El mini-portátil ha ayudado a fomentar dicho aprendizaje.

- $\quad$ Aprendizaje colaborativo: Internet, mini-portátiles y las herramientas comunicativas trabajadas en el aula (Edmodo, Eleven...) hacen que dicha estrategia haya aumentado de manera considerable en los centros.

- Aprendizaje personalizado: posibilidad de que el alumnado vaya a su propio ritmo de aprendizaje.

- Cambio de roles en el alumnado y el profesorado.

Dichas estrategias curriculares de integración de los medios didácticos se relacionan con la perspectiva técnica y práctica (Gallego ,2005). Por una parte, nos encontramos con un profesorado transmisor, un rol pasivo del alumnado y unos medios ajenos al contexto escolar. Frente a dicha perspectiva técnica, también encontramos usos prácticos y situacionales, un rol más activo del alumnado y unos medios más acordes con el contexto escolar que se nos presenta. Hablamos de la perspectiva práctica.

Se ha podido observar diferentes estrategias con respecto a los diferentes enfoques de aprendizaje centrados en la actividad constructiva del alumnado. Se relacionan con dos de las teorías propuestas por Coll, Mauri y Onrubia (2009): una concepción centrada en el aprendizaje del alumnado entendido como resultante de procesos psicológicos de naturaleza diversa y una concepción centrada en el aprendizaje como resultado de un proceso de naturaleza social. La primera concepción se relaciona con el aprendizaje autónomo y personalizado, pues se considera que el alumnado difiere en su aprendizaje y que, por lo tanto, la instrucción debe ser individualizada. La segunda concepción se relaciona con el aprendizaje colaborativo, la cual sitúa los procesos de enseñanza/aprendizaje en la actividad colaborativa o en la interactividad.

También se relacionada con tres de las etapas de aprendizaje con TIC propuestas por Benito (2009): enseñanza basada en Internet, el aprendizaje flexible y las redes sociales.

A lo largo de dicho estudio hemos podido observar que la llegada de los recursos proporcionados por el Programa Escuela 2.0 /REDXXI ha hecho replantearse la forma de trabajar a todo el profesorado participante. El uso de la PDI y los mini-portátiles han configurado un espacio distinto al tradicional, permitiendo establecer diferentes estrategias didácticas, fomentando aprendizajes basados en la autonomía y la colaboración. Por otra parte, se destaca el uso de las herramientas WEB 2.0 por parte del profesorado y el alumnado, ha permitido establecer una comunicación alumnadoprofesorado y alumnado-alumnado que trasciende las propias paredes de las aulas de los centros educativos.

En definitiva, aun entendiendo que los recursos o herramientas no proporcionan un cambio metodológico, sino que depende las estrategias y usos didácticos que realice el docente, sí que entendemos que la incorporación de dichos recursos en las clases de los centros educativos, han supuesto que el profesorado se replantee sus prácticas de aula. 


\section{Referencias}

Area, M (2008). La innovación pedagógica con TIC y el desarrollo de las competencias informacionales y digitales. Investigación en la escuela, (64), 5 18. Recuperado en http://www.investigacionenlaescuela.es/arti culos/64/R64_1.pdf

Area, M. y Pessoa, T. (2012). De lo sólido a lo líquido: Las nuevas alfabetizaciones ante los cambios culturales de la Web 2.0. Comunicar, 19 (38), 13-20. Recuperado en https://www.redalyc.org/pdf/158/158230830 03.pdf

Area, M., Alonso, C., Correa, JM., Moral, M., De Pablos, J., Paredes, J., Peirats, J., Sanabria, AL., San Martín, A. y Valverde, J. (2014). Las políticas educativas TIC en España después del Programa Escuela 2.0: las tendencias que emergen. RELATEC: Revista Latinoamericana de Tecnología Educativa, 13 (2), 11-34. Recuperado de https://relatec.unex.es/article/view/1473

Benito, M. (2009). Desafíos pedagógicos de la escuela virtual. Las TIC y los nuevos paradigmas educativos. Revista Telos, (78), 63-77 Recuperado en: https://telos.fundaciontelefonica.com/telos/ articulocuaderno.asp@idarticulo=2\&rev=78 .htm

Coll, C., Mauri, T. y Onrubia, J. (2009). Hacia una modelización del proceso de enseñanza y aprendizaje basado en las TIC. Teorías y enfoques centrados en la actividad constructiva del alumnado. En J. De Pablos (coord.), La formación del profesorado en la era de Internet (pp.145-162). Málaga: Aljibe.
De La Torre, A (2006). WEB educativa 2.0. Edutec: revista electrónica de tecnología educativa, (20). Recuperado en: http://edutec.rediris.es/Revelec2/revelec20/ anibal20.pdf

Gallego, M.J. (2005). La integración de las TIC en el currículo y en la organización escolar. En M. Cebrián de la Serna (coord.) Tecnologías de la información y la comunicación para docentes (pp.27-39). Madrid: Pirámide.

García Aretio, L. (2014): Web 2.0 vs web 1.0. Contextos Universitarios Mediados, $\mathrm{n}^{\circ} 14,1$ (ISSN: 2340-552X). Recuperado de http://espacio.uned.es/fez/eserv/bibliuned:UNESC O-contextosuniversitariosmediados14 1/Documento.pdf

Hargreaves, D. H. (1999). The knowledgecreating school. British journal of educational studies, 47(2), 122-144.

McMillan, J. y Schumacher, S. (2005). Investigación educativa. Madrid: Pearson. (5aed.).

Official Journal of the European Union (2006). Recommendation of the European Union and of the Council of 18 December 2006 on key competences for lifelong Learning (2006/962/EC). Recuperado en http://eurlex.europa.eu/LexUriServ/LexUriS erv.do?uri=OJ:L:2006:394:0010:0018:en:P DF

Simons, H. (2011). El estudio de caso: teoría y práctica. Madrid: Morata.

Stake, R. (1999). Investigación con estudio de casos. Madrid: Morata. 